\title{
Design of a Polishing Tool for Collaborative Robotics Using Minimum Viable Product Approach
}

\author{
Carlos Perez-Vidal ${ }^{\mathrm{a}}$, Luis Gracia ${ }^{\mathrm{b} *}$, \\ Samuel Sanchez-Caballero ${ }^{\mathrm{b}}$, J. Ernesto Solanes ${ }^{\mathrm{b}}$, Alessandro Saccon ${ }^{\mathrm{c}}$ and Josep Tornero ${ }^{\mathrm{b}}$ \\ ${ }^{a}$ Miguel Hernandez University, Avda. de la Universidad S/N, 03202 Elche (Spain); \\ ${ }^{b}$ Instituto IDF, Universitat Politècnica de València, Camino de Vera S/N, 46022 Valencia (Spain); \\ ${ }^{c}$ Department of Mechanical Engineering, Eindhoven University of Technology (TU/e), $5600 \mathrm{MB}$ \\ Eindhoven (The Netherlands).
}

(Received 14 September 2018; accepted 06 June 2019)

\begin{abstract}
A collaborative tool for robotic polishing is developed in this work in order to allow the simultaneous operation of the robot system and human operator to cooperatively carry out the polishing task. For this purpose, the collaborative environment is detailed and the polishing application is designed. Moreover, the polishing tool is developed and its implementation using the minimum viable product approach is obtained. Furthermore, a robust hybrid position-force control is proposed to use the developed tool attached to a robot system and some experiments are given to show its performance.
\end{abstract}

Keywords: Polishing tool; Cooperative polishing; Human-robot cooperation.

\section{Introduction}

Polishing processes represent the final surface treatment in several precision industries such as mould manufacturing, leather finishing and body car production. These processes are used to remove imperfections of the surface and improve its smoothness and brightness. In the case of automotive industry, the state of the art in body car defect detection has made a big progress during the last years (Alders, Lehe, and Wan 2001), but polishing process is still performed by hand.

After detecting and locating the imperfection, it must be polished, which is currently carried out by human operators, requiring skillful training, experience and dexterity. The work environment to which the human operator is exposed requires special equipment such as breathing masks for dust particles and hearing protection to reduce the noise level. In addition, exposure to the vibrations generated by this type of activity during long periods of time can cause nervous dysfunction, muscle-skeletal problems and vascular disorders (Vihlborg et al. 2017). Automating the polishing process would improve efficiency and could reduce these problems.

Several works related with non-collaborative robotized polishing or deburring of complex surfaces can be found. For instance, Mohammad, Hong, and Wang (2018) specially focused on tool design, whereas Nagata et al. (2007) tackled the problem using the CAD/CAM information of the surface. In Oba and Yamada (2017), a tool mounted over a parallel robot for the automotive industry is presented. Another approach is shown in Kalt, Monfared, and Jackson (2016), where the polisher is static and the robot moves the element to be polished. But till the moment, none of them has been

\footnotetext{
*Corresponding author. Email: luigraca@isa.upv.es
} 


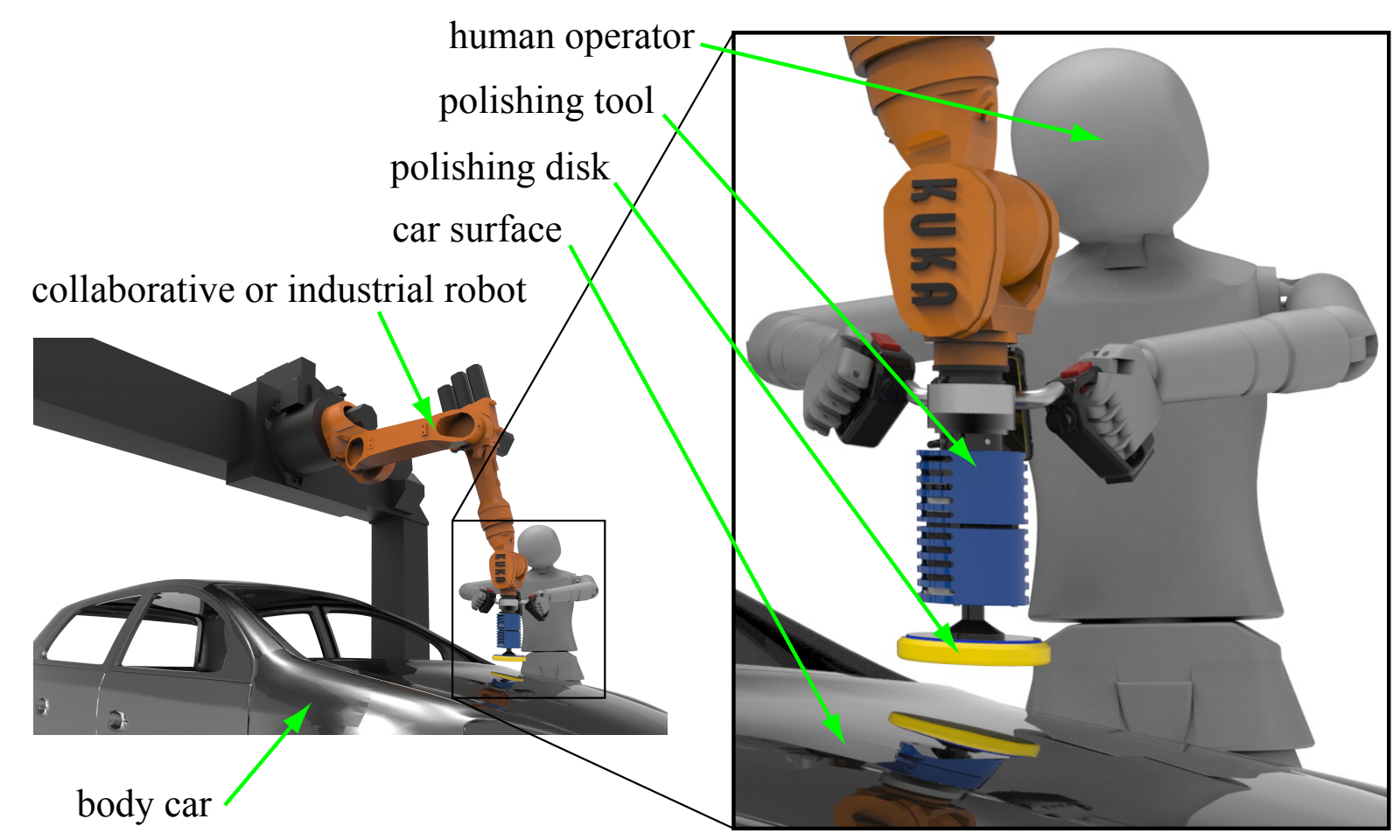

Figure 1.: Teaching by demonstration programming of a collaborative robot to perform polishing tasks.

successfully implemented in a real industrial process, which is mainly due to the great complexity involved in the automation of this task. On the one hand, many considerations such as trajectory tracking, tool orientation and applied force must be considered. On the other hand, the system must perform the task getting feedback from the surface based on computer vision and/or tactile inspections. Hence, the development of an autonomous polishing system requires flexibility and adaptability, skills inherent to a human operator and really difficult to provide to a robot.

This work proposes the use of robot systems to help human operators in polishing tasks instead of replacing them, since operators are essential due to the dexterity needed in the task (Surdilovic and Schreck 2010). As long as operators and robots have to share the same workspace, collaborative robots have been developed to be safer than traditional industrial robots. This is the case of robots such as Baxter and Sawyer (Fitzgerald 2013) by Rethink Robotiks, LBR iiwa (Shepherd and Buchstab 2014) by Kuka, UR3/5/10 (Hawkins, Bansal, and Bobick 2013) by Universal Robots and YuMi (Kirschner et al. 2016) by ABB, amongst others.

All these collaborative robots can be programmed in a safe way using the so-called "teaching by demonstration", which offers an intuitive way of programming instead of the classic programming method. The final system could work in two phases: teaching phase, in which the operator performs the task with the robot in "recording mode"; and execution phase, in which the tool reproduces the same trajectory applying a constant force against the surface and keeping perpendicularity all the time. This concept can be seen in Fig. 1, where a human operator is teaching the robot the polishing task and, subsequently, the robot performs it autonomously.

This work develops a collaborative tool for the polishing task mentioned above. An implementation of the polishing tool is required to test its performance and validate or modify its design parameters in order to move towards the implementation of an industrial solution. The implementation presented in this work is based on the minimum viable product (MVP) criteria, which simplifies the product to the minimum required features and is used to validate the assumptions 
made and to evaluate its performance.

The paper is organized as follows. Section 2 presents the collaborative environment and polishing application proposed in this work, whereas Section 3 develops the design of the proposed polishing tool and its implementation using the minimum viable product approach. Then, a robust hybrid position-force control is proposed in Section 4 to use the developed tool attached to a robot system, where some experiments are included. Finally, some discussion is presented in Section 5, whereas some concluding remarks are given in Section 6.

\section{Design of the collaborative environment and task procedure description}

In this section, a collaborative environment is designed for the body car industry, including the description of the collaborative polishing procedure to remove the paint defects and imperfections on the body car surface.

The body car production is usually carried out as a set of tasks performed by three different production departments: stamping, cutting and assembling. The stamping department provides the steel or aluminum sheets shaped by a press machine. The cutting department refines the shapes through three-dimensional cut processes usually performed by industrial robots. The assembly department puts together the complete steel or aluminum pieces that will be mounted on the frame of the car to build the body of the car that will be painted. The last part of this production line can be seen in Fig. 2, where the layout of the production plant is depicted.

Assembled elements are controlled in the geometry and aesthetic departments, by human operators or by automatic machines depending on the car production volumes. Surface quality is checked analyzing the deformation of different areas of the body car under special lighting patterns. This process can be performed using inspection tunnels (Arnal et al. 2017), like the one represented in Fig. 2c. When the body car passes through the detection tunnel, the computer vision system checks the quality of the whole surface. In case of detecting a damage, the system provides the information to a human operator equipped with Virtual Reality (VR) glasses (see Fig. 2d and Fig. 2e) and to a robot system equipped with a polishing tool (see Fig. 2b and Fig. 2e).

The human worker gets the task information through the VR glasses, including the position, type of surface damage and procedure to eliminate it (usually) by polishing. Some of these damages or imperfections on the body car surface are treated by the robot equipped with the collaborative polishing tool developed in this work. The robot is able to perform the polishing task autonomously or cooperatively with the human operator, alone in the workspace or sharing it with people. The operator gets the information about the whole process through his VR glasses and has to approve the quality level of the task performed by the robot. This information is used to train an expert system that improves the next polishing task by learning from the expert inputs. The process proposed in this work is visually depicted in Fig. 2a, where the factory layout can be seen.

The collaborative polishing task performed by the robot could be carried out implementing the flow chart shown in Fig. 3. This scheme depicts a set of steps to be followed once the task has started. A hierarchical selector can choose between two Grafcet macro-steps (Julius et al. 2017) devoted to perform the polishing (left branch in Fig. 3) and finishing the task (right branch in Fig. 3). The polishing macro-step begins with the tool approach. It can be performed manually by the operator or automatically considering the knowledge of the body car CAD model or using some kind of distance sensor (infrared, ultrasonic, 3D vision system, etc.). Once the collaborative tool contacts the car surface, the automatic polishing starts and the system remains in this state until the human operator interacts with the tool. In this case, the system switches to the collaborative mode control, i.e., the operator guides the robot tool, and resumes the automatic polishing when the operator releases the tool. 


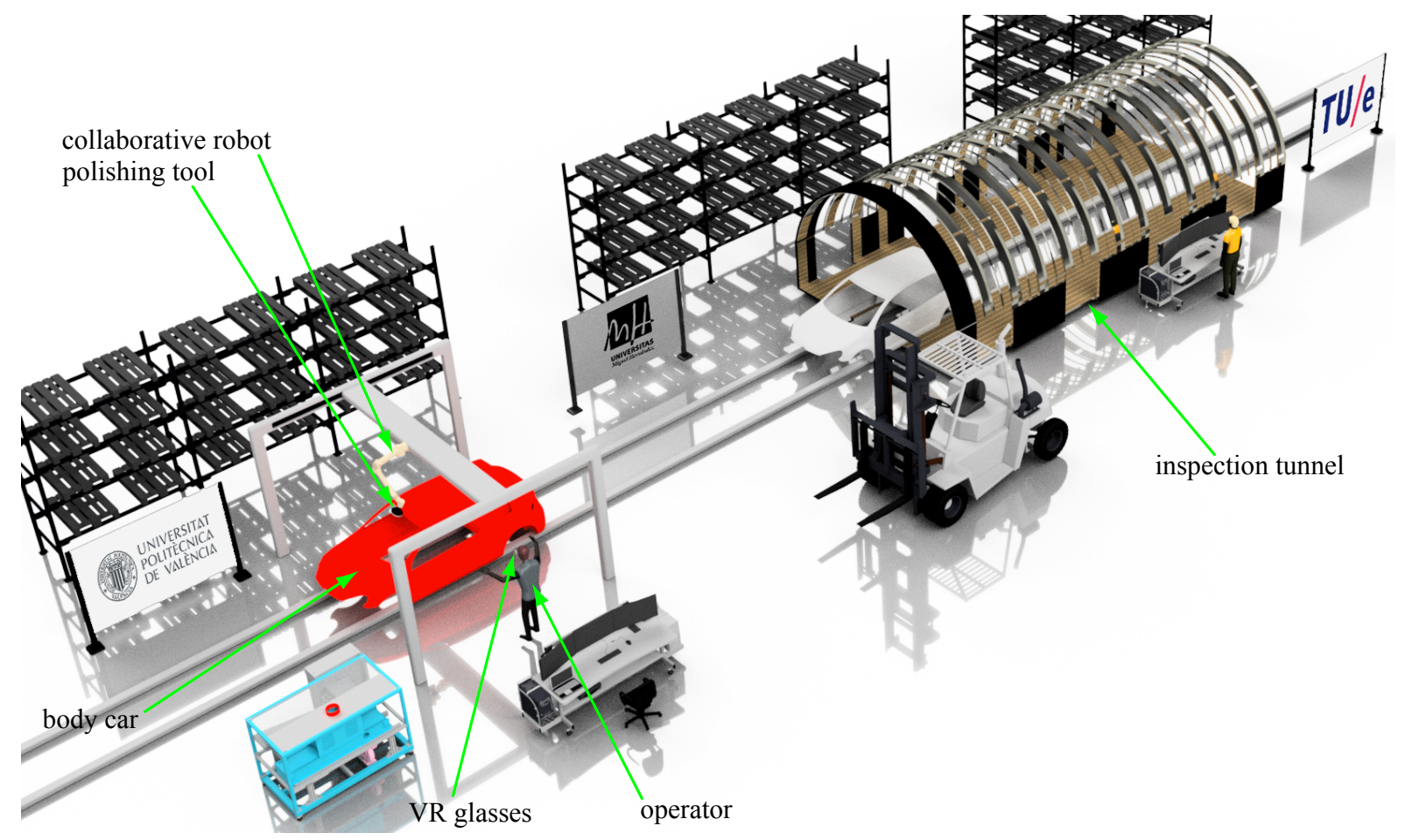

(a) Production plant layout considering automatic inspection and robotic polishing

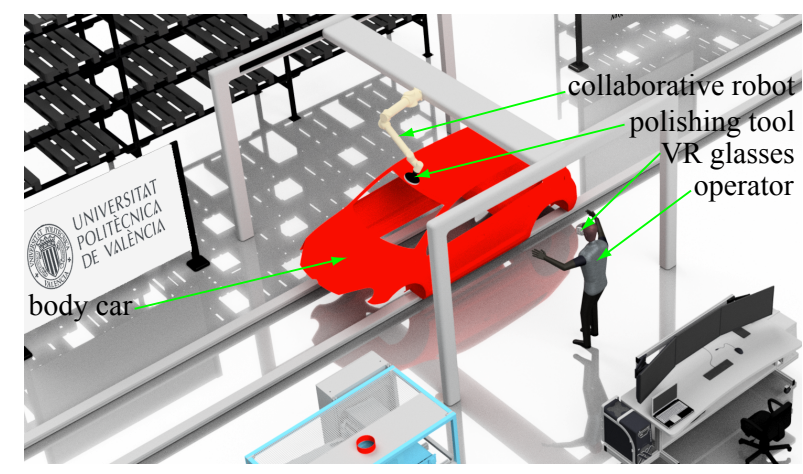

(b) Polishing area of the body car production plant

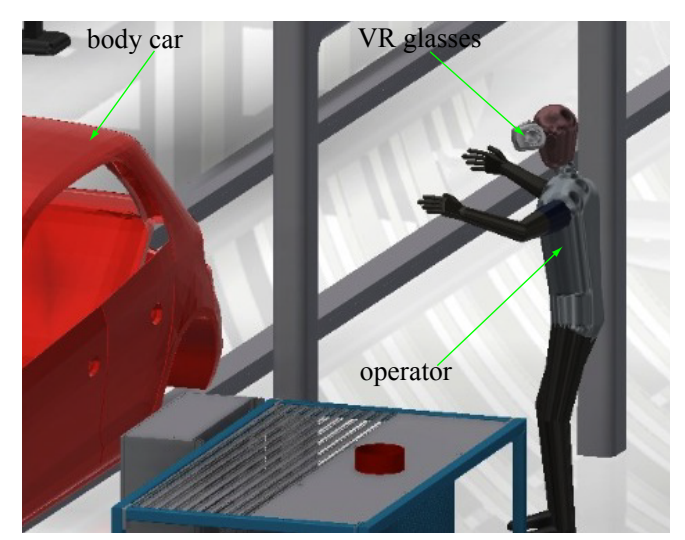

(d) The system includes virtual reality and augmented reality to improve the human operator performance

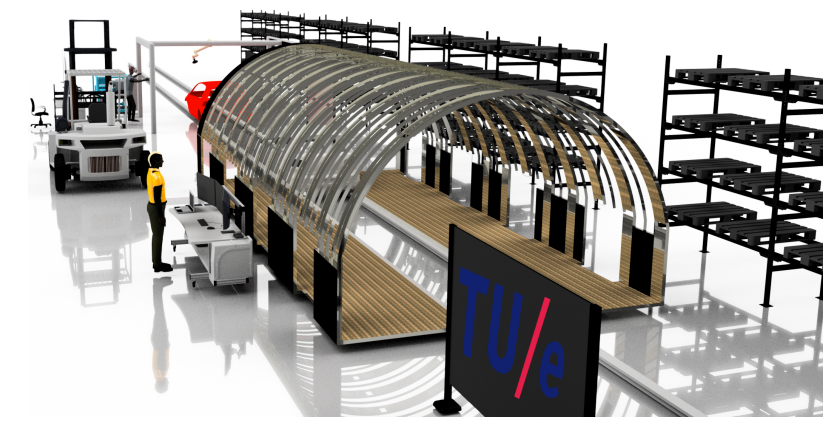

(c) Defect location is performed by inspection tunnels equipped with computer vision

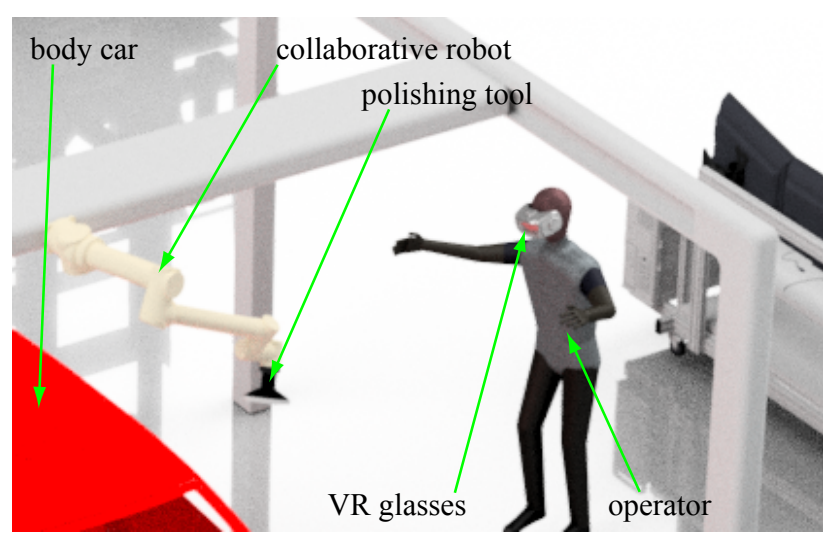

(e) The operator gets the information of the process through VR glasses

Figure 2.: Collaborative environment in a factory layout. 


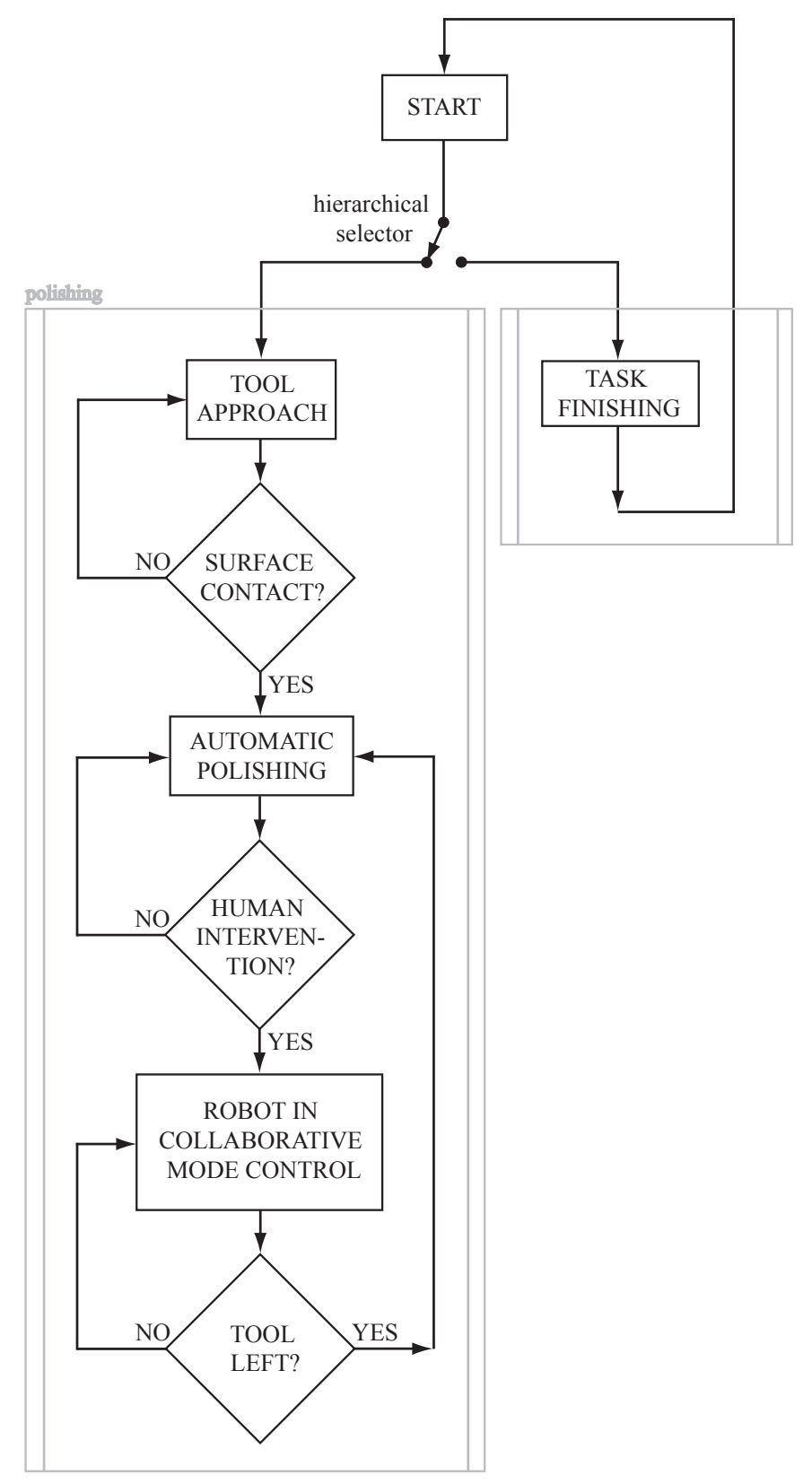

Figure 3.: Flow chart of the collaborative polishing task.

\section{Design of the polishing tool and MVP}

On the one hand, as discussed in the Introduction section, several solution solutions can be found in the literature to solve the problem of surface treatment using robots, e.g., see the works of Mohammad, Hong, and Wang (2018), Nagata et al. (2007), Oba and Yamada (2017), and Kalt, Monfared, and Jackson (2016), amongst others. On the other hand, the robot motion guidance problem through force sensors that evaluate the interaction of the human has been widely tackled, e.g., see the works of Dimeas and Aspragathos (2016), Khan et al. (2017) and Vogel et al. (2015), amongst others. But the synergistic or symbiotic combination (Symplexity 2015) of both solutions, robotic surface treatment and robot motion guidance, is uncommon in published literature 
as discussed below.

On the one hand, due to the complexity of the shape of the product surfaces, human operators have difficulties in maintaining a constant pressure and tool perpendicularity to the surface at all times. On the other hand, when the product to be treated is part of a production line where it is in motion or its morphology is constantly changing, it is difficult for robots to carry out automatic treatment operations efficiently. Hence, to mitigate the drawbacks of both, manual and robotic automatic surface treatment, this work proposes a flexible surface treatment solution which adopts the form of a human operator performing the task of "guiding" the tool along the object surface, while the robot manipulator is in charge of automatically maintaining both the tool's pressure on the surface and the tool's perpendicularity to the surface. After this teaching phase, the robot can automatically reproduce the whole operation using the trajectory "learned" from the human operator.

For this purpose, tools like those in Mohammad, Hong, and Wang (2018) or in Oba and Yamada (2017) could be adapted to obtain a collaborative tool by performing a few changes. Based on this idea, a prototype of the tool has been designed as shown in Fig. 4. The most important elements of the system are the following: an element that is capable of autonomously generate a relative trajectory between the polisher and the surface (serial robot); an abrasive disk with a turning movement; a human-machine interface (HMI) for the interaction with the polishing system that includes a pair of handlers; a two degrees of freedom mechanical device to increase the system ergonomics (Alexopoulos, Mavrikios, and Chryssolouris 2018); and a force-torque sensor to obtain the interaction with the environment (surface and operator).

The configuration chosen in this case is similar to that in Mohammad, Hong, and Wang (2018), where the polisher has been attached to the robot's end-effector and it performs the relative movement with the surface. The polishing tool proposed in this work is equipped with a 500 watts brush motor, transmission elements, and a quick change hex shank head for easily change the polishing disks. An important element of the tool is the handle and its base. This base incorporates the force-torque sensor and a two degrees of freedom mechanical element to allow rotation in two axis (see Fig. 4c and Fig. 4d) in order to increase the ergonomics of the system. Its movement is passive and has been implemented with electrically actuated lockers operated by the worker, allowing blocking and unlocking the movement by the operator. This base allows for polishing in a comfortable way both vertical surfaces and horizontal surfaces. The system also incorporates a touch screen panel that completes the interaction capabilities of the tool. The HMI is made up by the handles and the touch screen panel, it is removable (see Fig. 4b) and it can be used to perform tasks related to programming, training and production.

A force-torque sensor, namely polishing sensor in Fig. 4a, is required to measure the forces and torques applied by the tool to the object being polished and those generated by the human operator. These measures allow to properly perform the polishing task, i.e., to control the pressure applied by the tool to the surface and its perpendicularity as well as to move the robot tool according to the forces applied by the human operator to the tool handles. It is worth noting that a second force sensor could be considered, either in the tool or in the robot end-effector, in order to measure independently both interaction forces, i.e., those between the tool and the object being polished and those between the tool and the human operator. This configuration would allow a more accurate measure of these interaction forces. As discussed below, this option is not considered here and remains as further work.

The concept of Minimum Viable Product (MVP) was introduced by Robinson (2001), and popularized by Ries (2009) and Blank (2010). In product development, the MVP is a product with enough features to satisfy the initial customer's requirements, providing feedback for future developments. Learning from an MVP is often less expensive and time-consuming than developing a product with more features, which increase costs and risks if the product fails, e.g., due to incorrect assumptions. Thus, once the MVP has been tested and approved, product enhancements can be made including more features. 


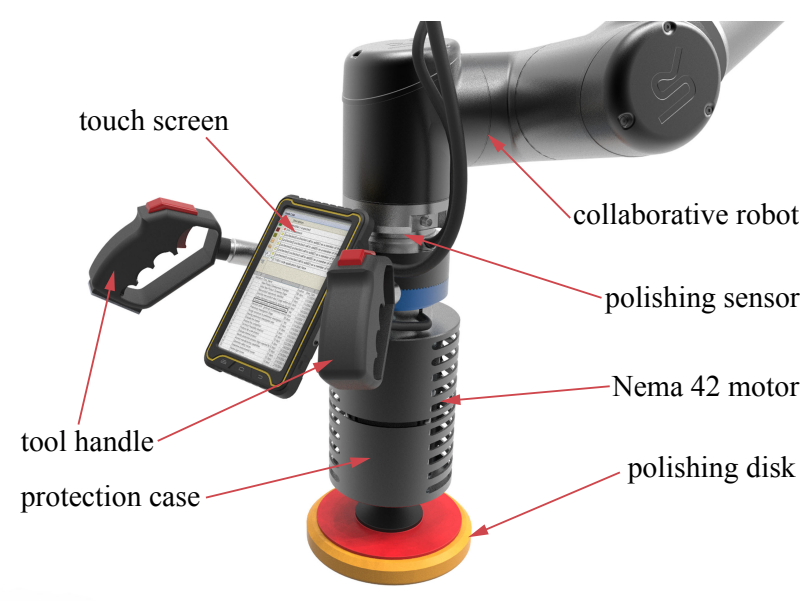

(a) Elements of the polishing tool

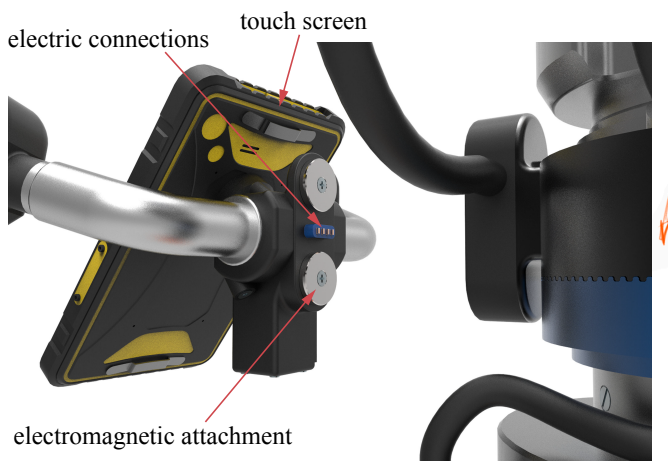

(b) Removable human machine interface

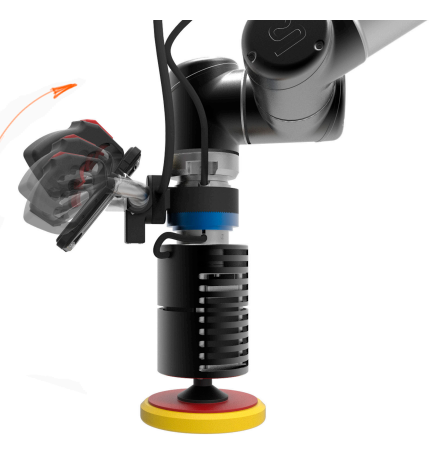

(c) Horizontal ergonomics enhancement

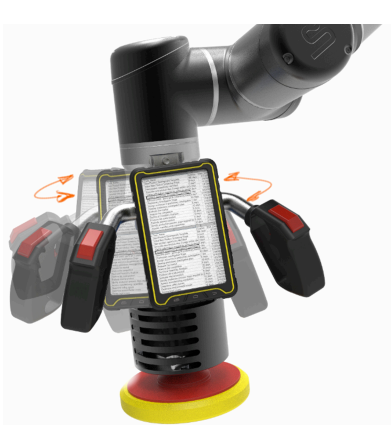

(d) Vertical ergonomics enhancement

Figure 4.: Design of the polishing tool with a description of the most relevant elements. Detachment of the human machine interface (HMI) and ergonomic considerations have been included in the design.

Therefore, this work develops an MVP of the polishing tool, focused on the essential features and removing the secondary or minor ones. In particular, the schematic representation MVP tool proposed in this work can be seen in Fig. 5. This MVP tool is implemented as shown in Fig. 6 . The force-torque sensor used in this work is the ATI FTN Gamma SI-130-10. Several material resistance tests have been performed with the MVP tool to verify that none of the mechanical elements of the polisher get broken or damaged. Details omitted for brevity.

It is worth noting that, although the proposed robot tool has been specifically designed for human-robot collaborative polishing, its kinematic calibration process can be performed using conventional calibration procedures, e.g., see Fassi et al. (2006) and Andres, Gracia, and Tornero (2011), amongst others.

\section{Robust hybrid position-force control}

\subsection{Overview of the control scheme}

The collaborative tool developed in this work can be attached to a robot system and a force control is required to allow the operation of the whole system. In particular, the robust hybrid position-force control algorithm presented in Gracia et al. (2018) can be used. This algorithm 


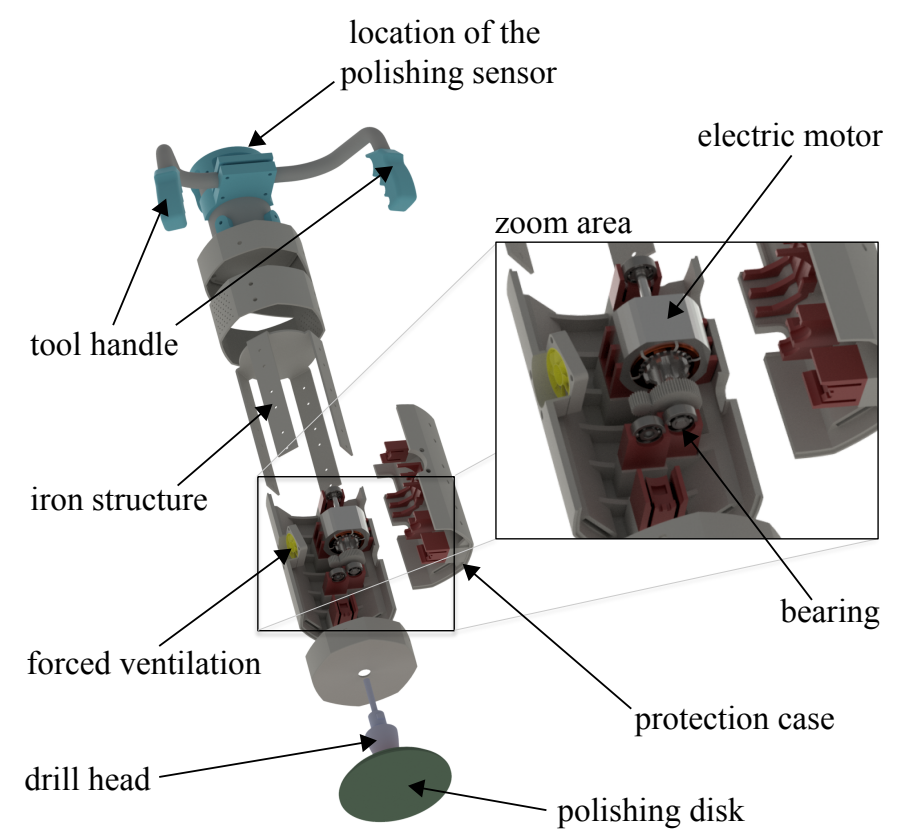

Figure 5.: MVP version of the polishing tool.

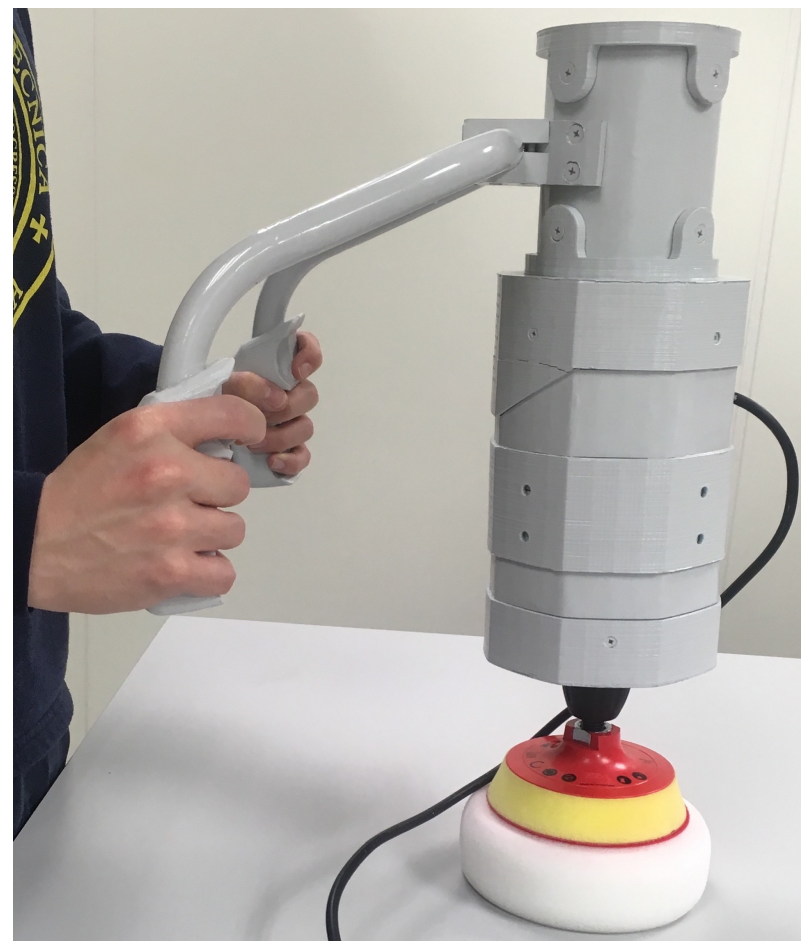

Figure 6.: Implementation of the MVP tool.

uses a task priority strategy (Nakamura, Hanafusa, and Yoshikawa 1987) and sliding mode control (SMC) (Gracia, Sala, and Garelli 2014) and can be adapted for the proposed polishing task as detailed below.

The proposed scheme for the robot control is shown in Fig. 7, where it can be seen that three prioritized levels are considered: the high-priority level (Level 1) is used to guarantee that the tool is perpendicular to the surface and that the pressure between the tool and the object is equal to the 


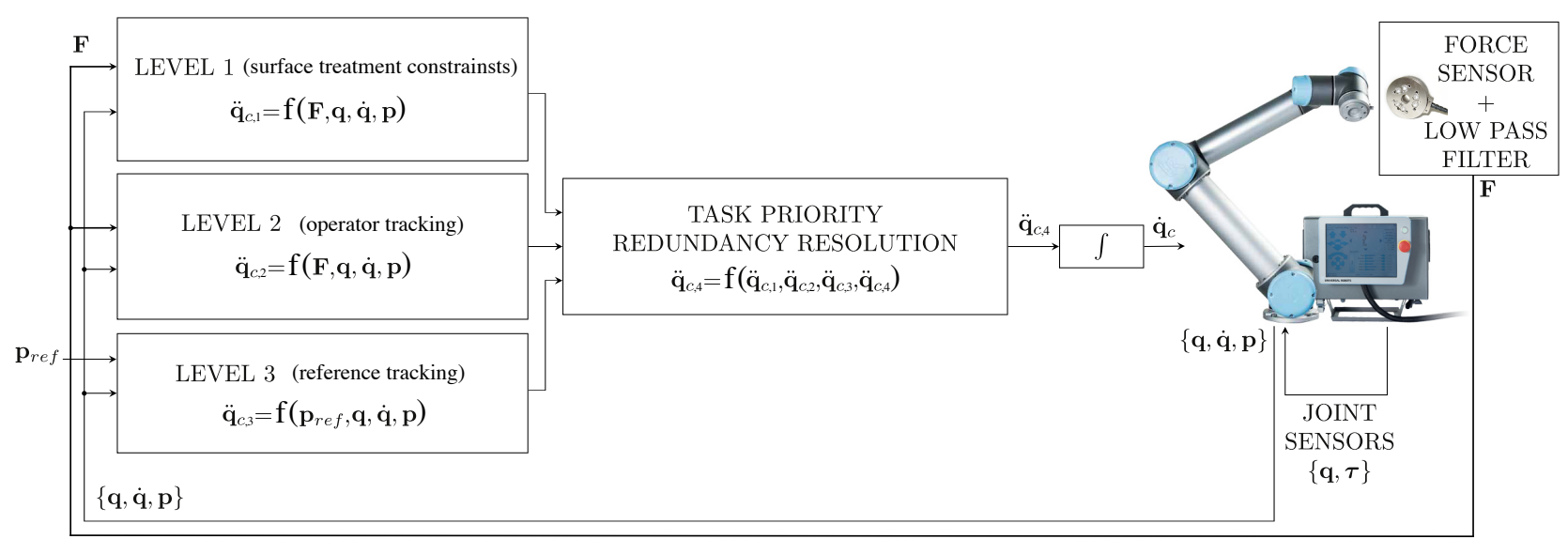

Figure 7.: Block diagram of the control system.

desired polishing force; the medium-priority level (Level 2) is used to track the human operator's movements; and, finally, the low-priority level (Level 3) is used to track a reference trajectory in order to apply the polishing on a specific area of the surface.

The input to these levels are: the robot state $\{\mathbf{q}, \dot{\mathbf{q}}\}$ and tool pose $\mathbf{p}$ obtained from the robot controller; the vector $\mathbf{F}$ of forces and torques measured by the polishing sensor located at the robot tool, which has already been filtered by the sensor electronics; and the reference $\mathbf{p}_{\text {ref }}$ for the tool pose. Each Level $i$ must satisfy an acceleration equality or control equation of the form $\mathbf{A}_{i} \ddot{\mathbf{q}}_{c}=\mathbf{b}_{i}$, where $\ddot{\mathbf{q}}_{c}$ represents the command for the joint acceleration vector, that is obtained using SMC in order to benefit from its inherent robustness. Furthermore, the square error of these control equations is minimized using the task priority redundancy resolution to obtain the commanded acceleration $\ddot{\mathbf{q}}_{c, 4}$, which is integrated and sent to the robot controller. Finally, the robot controller performs a low-level control loop to track the commanded velocity $\dot{\mathbf{q}}_{c}$ using the current angles $\mathbf{q}$ and torques $\tau$ measured by the joint sensors.

\subsection{Control equations}

\subsubsection{Constraints}

Each Level $i$ is designed to fulfill a number of equality constraints $\sigma_{i, j}=0$ or inequality constraints $\sigma_{i, j} \leq 0$, where $\sigma_{i, j}$ is the so-called constraint function of the $j$-th constraint of Level $i$. These constraints will be fulfilled using SMC (Gracia, Sala, and Garelli 2014). Moreover, the actual constraint function $\sigma_{i, j}$ is modified as follows to smooth the switching behavior of the SMC:

$$
\phi_{i, j}=\sigma_{i, j}+K_{i, j} \dot{\sigma}_{i, j},
$$

where $\phi_{i, j}$ is the modified constraint function and $K_{i, j}$ is the smoothing parameter for the $j$-th constraint of Level $i$.

On the one hand, the constraints for the first two levels are defined below depending on the vector $\mathbf{F}=\left[\begin{array}{llllll}F_{x} & F_{y} & F_{z} & F_{\alpha} & F_{\beta} & F_{\gamma}\end{array}\right]^{\mathrm{T}}$ of forces and torques measured by the polishing sensor, where all six coordinates are relative to the tool coordinate system. On the other hand, the constraints for the third level is defined below depending on the reference $\mathbf{p}_{r e f}$ for the tool pose. 


\subsubsection{Level 1}

Three equality constraints are defined in Level 1 for the polishing surface treatment as follows:

$$
\begin{aligned}
& \sigma_{1, z}=F_{z}-F_{z, r e f}=0 \\
& \sigma_{1, \alpha}=F_{\alpha}=0 \\
& \sigma_{1, \beta}=F_{\beta}=0,
\end{aligned}
$$

where $F_{z}$ is the linear force measured by the polishing sensor in the tool $Z$-axis, $F_{\alpha}$ and $F_{\beta}$ are the torques measured by the polishing sensor in the tool $X$-and $Y$-axes, and $F_{z, \text { ref }}$ is the desired force between the tool and the surface being treated in the tool $Z$-axis. Hence, the first constraint is used to attain the desired force $F_{z, r e f}$ between the tool and the surface, whereas the last two constraints are used to keep the tool orientation perpendicular to the surface, since the torques in $X$ - and $Y$-axes are zero if the tool is perfectly perpendicular to the surface.

As mentioned above, constraints (2)-(4) are fulfilled using SMC, yielding the following control equation:

$$
\mathbf{A}_{1} \ddot{\mathbf{q}}_{c}=\mathbf{b}_{1}
$$

where $\mathbf{A}_{1}$ and $\mathbf{b}_{1}$ are the matrix and vector established by the SMC to fulfill the constraints of Level 1, see Gracia et al. (2018) for further details.

\subsubsection{Level 2}

The following inequality constraint is considered in Level 2 to track the human operator's forces:

$$
\sigma_{2, x y}=\sqrt{F_{x}^{2}+F_{y}^{2}}-F_{x y, 0}=F_{x y}-F_{x y, 0} \leq 0,
$$

where $F_{x}$ and $F_{y}$ are the linear forces detected by the polishing sensor in its $X$ - and $Y$-axes, which are perpendicular to the robot end-effector and are mainly due to the human operator's forces, $F_{x y}$ is the magnitude of these linear forces and $F_{x y, 0}$ is a threshold so that the constraint becomes active when the magnitude $F_{x y}$ is larger than this threshold, in which case the robot tool is moved by the proposed SMC in the direction of the detected forces to fulfill the constraint.

Hence, when the human operator pushes the tool handles the force $F_{x y}$ detected by the sensor exceeds the threshold $F_{x y, 0}$, the inequality constraint (6) becomes active and the robot control changes its behavior, i.e., the tool movement on the surface "switches" from tracking the reference trajectory (Level 3 or low-priority level) to tracking the operator forces (Level 2 or medium-priority level), yielding deviations from the reference trajectory.

As before, constraint (6) is fulfilled using SMC, yielding the following control equation:

$$
\mathbf{A}_{2} \ddot{\mathbf{q}}_{c}=\mathbf{b}_{2}
$$

where $\mathbf{A}_{2}$ and $\mathbf{b}_{2}$ are the matrix and vector established by the SMC to fulfill the constraint of Level 2, see Gracia et al. (2018) for further details.

\subsubsection{Level 3}

The following equality constraint is considered in Level 3 for reference tracking:

$$
\sigma_{3, e}=\mathbf{e}=\mathbf{p}_{r e f}-\mathbf{p}=0
$$


where $\mathbf{e}$ is the tool pose error, yielding the following control equation:

$$
\mathbf{A}_{3} \ddot{\mathbf{q}}_{c}=\mathbf{b}_{3},
$$

where $\mathbf{A}_{3}$ and $\mathbf{b}_{3}$ are the matrix and vector established by the SMC to fulfill the constraint of Level 3, see Gracia et al. (2018) for further details.

\subsubsection{Redundant robots}

In case of redundant robots (Gracia, Sala, and Garelli 2012), another level with the lowest-priority can be considered to use the additional degrees of freedom of the robot, yielding, in general, the control equation:

$$
\mathbf{A}_{4} \ddot{\mathbf{q}}_{c}=\mathbf{b}_{4} .
$$

where $\mathbf{A}_{4}$ and $\mathbf{b}_{4}$ are the matrix and vector for the control equation of Level 4 .

\subsubsection{Commanded acceleration}

The task-priority redundancy resolution (Nakamura, Hanafusa, and Yoshikawa 1987) is used to hierarchically minimize the square error of the control equations (5),(7),(9) and (10). In particular, the recursive solution for the commanded joint acceleration is given by (Siciliano and Slotine 1991):

$$
\begin{aligned}
\ddot{\mathbf{q}}_{c, 1} & =\mathbf{A}_{1}^{\dagger} \mathbf{b}_{1} \\
\mathbf{N}_{1} & =\mathbf{I}-\mathbf{A}_{1}^{\dagger} \mathbf{A}_{1} \\
\ddot{\mathbf{q}}_{c, 2} & =\ddot{\mathbf{q}}_{c, 1}+\left(\mathbf{A}_{2} \mathbf{N}_{1}\right)^{\dagger}\left(\mathbf{b}_{2}-\mathbf{A}_{2} \ddot{\mathbf{q}}_{c, 1}\right) \\
\mathbf{N}_{2} & =\mathbf{N}_{1}\left(\mathbf{I}-\left(\mathbf{A}_{2} \mathbf{N}_{1}\right)^{\dagger}\left(\mathbf{A}_{2} \mathbf{N}_{1}\right)\right) \\
\ddot{\mathbf{q}}_{c, 3} & =\ddot{\mathbf{q}}_{c, 2}+\left(\mathbf{A}_{3} \mathbf{N}_{2}\right)^{\dagger}\left(\mathbf{b}_{3}-\mathbf{A}_{3} \ddot{\mathbf{q}}_{c, 2}\right) \\
\mathbf{N}_{3} & =\mathbf{N}_{2}\left(\mathbf{I}-\left(\mathbf{A}_{3} \mathbf{N}_{2}\right)^{\dagger}\left(\mathbf{A}_{3} \mathbf{N}_{2}\right)\right) \\
\ddot{\mathbf{q}}_{c, 4} & =\ddot{\mathbf{q}}_{c, 3}+\left(\mathbf{A}_{4} \mathbf{N}_{3}\right)^{\dagger}\left(\mathbf{b}_{4}-\mathbf{A}_{4} \ddot{\mathbf{q}}_{c, 3}\right)
\end{aligned}
$$

where I denotes the identity matrix, superscript $\uparrow$ denotes the Moore-Penrose pseudoinverse (Golub and Van Loan 1996) and $\ddot{\mathbf{q}}_{c, i}$ and $\mathbf{N}_{i}$ are the solution vector and null-space projection matrix for the set of first $i$ levels. Therefore, the commanded acceleration $\ddot{\mathbf{q}}_{c, 4}$ is integrated and sent to the robot controller, as indicated above.

\subsection{Experiments}

The proposed robot control was tested in a collaborative robot for polishing a horizontal flat surface, where a circular reference trajectory was considered. The trajectories, forces-torques and control actions obtained in this test can be seen in Fig. 8, Fig. 9 and Fig. 10, respectively.

In particular, Fig. 8 shows the circular reference trajectory (thick line) and the real robot trajectory (thin line), where it can be seen that the robot tracks the circular reference trajectory while performing the polishing task except during the maneuvering process of the human operator. In this sense, the bottom plot of Fig. 9 shows signal $\sigma_{2, x y}$, which is related to the force exerted by the operator in the plane perpendicular to the robot end-effector, where it can be seen that the operator maneuvering process starts at around 20s and ends at around 35s. Hence, when the 


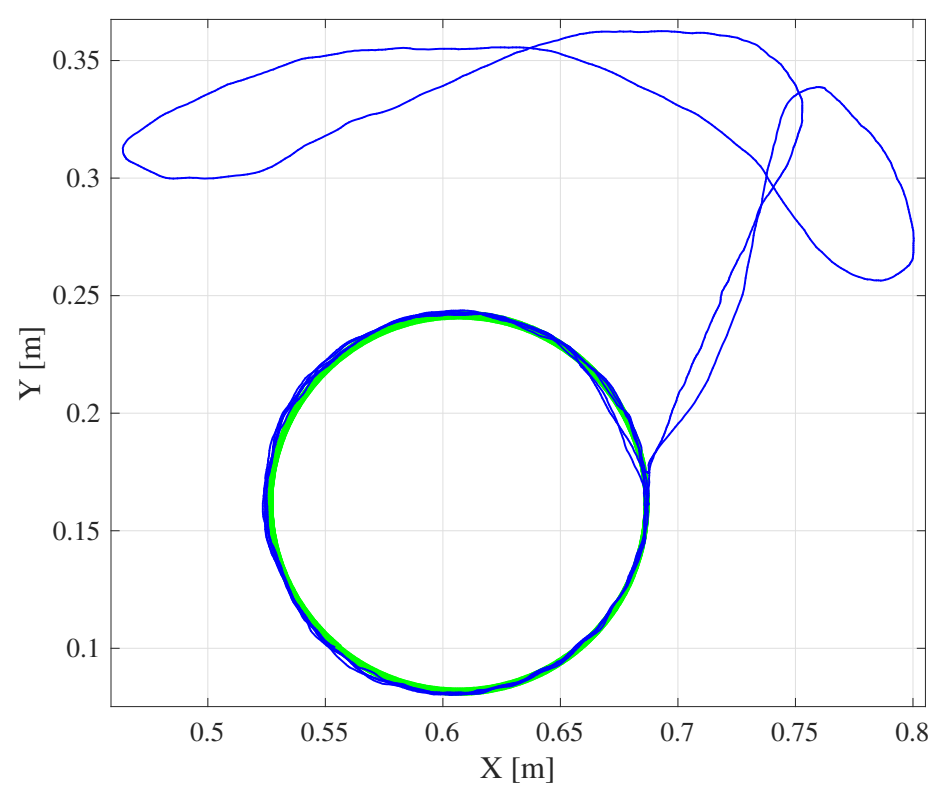

Figure 8.: Reference trajectory (thick line) and trajectory followed by the robot end-effector (thin line).
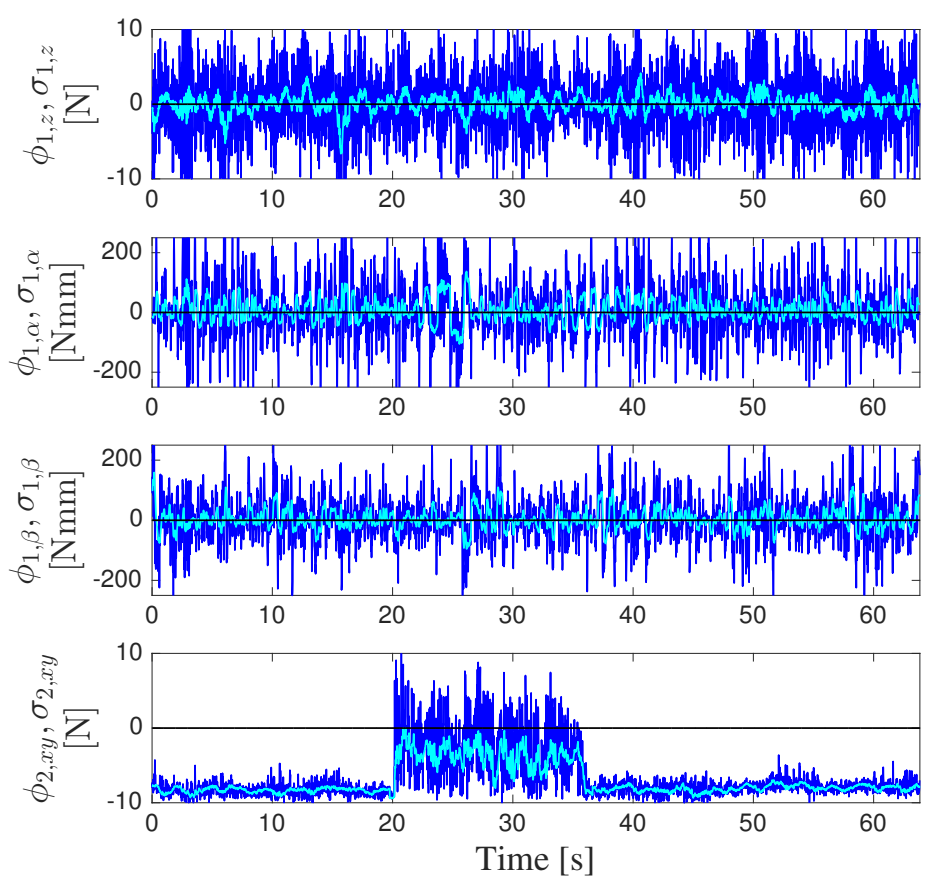

Figure 9.: Forces and torques measured by the polishing sensor.

operator starts pushing the robot handles at 20s the robot abandons the tracking of the circular reference trajectory and resumes it when the operator releases the handles at $35 \mathrm{~s}$.

The first three plots of Fig. 9 show signal $\sigma_{1, z}$, which is the difference between the desired and actual polishing force between the robot end-effector and the surface, see (2), and signals $\sigma_{1, \alpha}$ and $\sigma_{1, \beta}$, which represent the torques generated by the surface when the robot end-effector is not flat to the surface. Note that all these signals are switching around zero, indicating that the polishing of the surface is being performed properly: the robot pressure on the surface is approximately equal to the desired polishing pressure and the orientation of the robot end-effector is kept perpendicular 

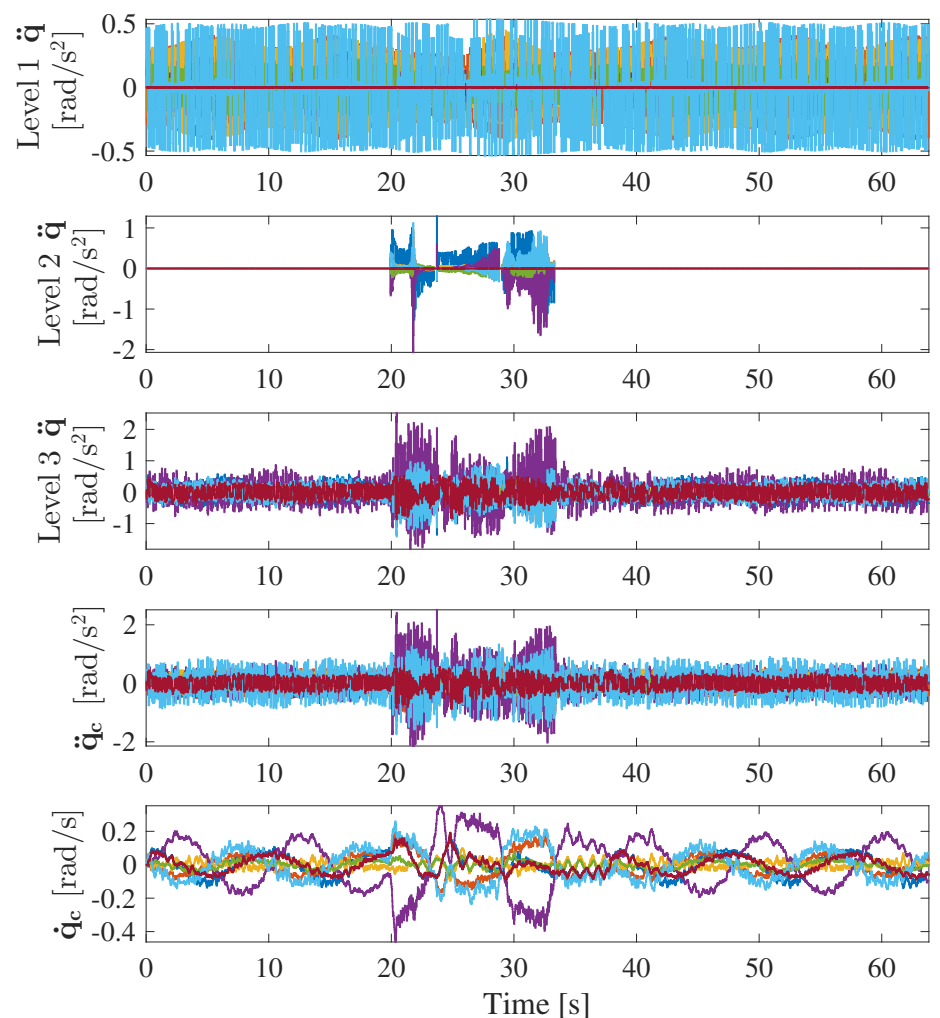

Figure 10.: Commanded actions generated by the robot control.

to the surface.

Fig. 10 shows the control commands computed during the experiment, where it can be seen that all three levels contribute to the commanded joint accelerations, although those for Level 1 are smaller, since only small acceleration corrections are needed to keep the right polishing pressure and robot orientation.

\section{Discussion}

The proposed robot control had a good performance in the above tests. However, these results are limited to flat or slightly curved horizontal surfaces. Therefore, further experiments are required to evaluate its behavior for other conditions: curved surfaces, non-horizontal surfaces, etc. It is interesting to remark that the convexity/concavity characteristics of the target object, i.e., the object to be polished, limits the size or diameter of the polishing disk in order to properly apply the polishing. Similarly, the size of the surface to be polished also limits the size of the polishing disk diameter. However, these issues also apply to manual polishing approaches.

Finally, in order to use the proposed robot control with the developed polishing tool, a redesign of the tool may be required in order to enhance its features, e.g., reducing the tool size, reducing the tool weight, increasing the tool resistance, etc. However, this issue remains as further work.

\section{Conclussion}

This works has been focused on the development and implementation of a novel collaborative tool for robotic polishing in order to allow the simultaneous operation of the robot system and operator 
to cooperatively carry out the polishing task. To the best of the authors' knowledge, this is the first robot tool designed for cooperative polishing. For this purpose, the collaborative environment and polishing application have been designed. Moreover, the polishing tool has been developed using the minimum viable product approach. Furthermore, a robust hybrid position-force control has been proposed to use the developed tool attached to a robot system and some experiments have been shown to illustrate its performance.

\section{Acknowledgements}

This work was supported in part by the Spanish Government under project DPI2017-87656-C2-1-R and the Generalitat Valenciana under Grant VALi+d APOSTD/2016/044.

\section{References}

Alders, K., M. Lehe, and G. Wan. 2001. "Method for the automatic recognition of surface defects in body shells and device for carrying out said method" US Patent 6,320,654, Nov 2001. URL https://www.google.ch/patents/US6320654.

Alexopoulos, K., D. Mavrikios, and G. Chryssolouris. 2018. "ErgoToolkit: an ergonomic analysis tool in a virtual manufacturing environment". Int. Journal of Computer Integrated Manufacturing 26 (5): 440-452.

Andres, J., L. Gracia, and J. Tornero. 2011. "Calibration and control of a redundant robotic workcell for milling tasks". International Journal of Computer Integrated Manufacturing 24 (6): 561-573.

Arnal, L., J.E. Solanes, J. Molina, and J. Tornero. 2017. "Detecting dings and dents on specular car body surfaces based on optical flow". Journal of Manufacturing Systems 45: 306-321.

Blank, S. 2010. "Perfection By Subtraction - The Minimum Feature Set". http://steveblank.com/2010/03/04/perfection-by-subtraction-the-minimum-feature-set/. (Accessed August, 2018).

Dimeas, F., and N. Aspragathos. 2016. "Online Stability in Human-Robot Cooperation with Admittance Control". IEEE Trasactions on Haptics 9: 267-278.

Fassi, I., G. Legnani, D. Tosi, A. Omodei, and L.K. Huat. 2006. "Calibration of serial manipulators: theory and applications". Industrial robotics: programming, simulation and applications 147-170.

Fitzgerald, C. Developing Baxter, A new industrial robot with common sense for U.S. manufacturing. 2013.

Golub, G., and C. Van Loan. 1996. "Matrix Computations". 3rd ed. Baltimore, MD: The Johns Hopkins University Press.

Gracia, L., A. Sala, and F. Garelli. 2012. "A supervisory loop approach to fulfill workspace constraints in redundant robots". Robotics and Autonomous Systems 60:1-15.

Gracia, L., A. Sala, and F. Garelli. 2014. "Robot coordination using task- priority and sliding-mode techniques". Robotics and Computer-Integrated Manufacturing 30: 74-89.

Gracia, L., J. E. Solanes, P. Munoz-Benavent, J. Valls-Miro, C. Perez-Vidal, and J. Tornero. 2018. "Adaptive Sliding Mode Control for Robotic Surface Treatment Using Force Feedback". Mechatronics 52: 102-118.

Hawkins, K.P., N. Vo, S. Bansal, and A. F. Bobick. 2013. "Probabilistic human action prediction and waitsensitive planning for responsive human-robot collaboration". 13th IEEE-RAS International Conference on Humanoid Robots (Humanoids). Atlanta, USA. October 15-17.

Julius, R., M. Schrenberg, F. Schumacher, and A. Fay. 2017. "Transformation of GRAFCET to PLC code including hierarchical structures". Control Engineering Practice 64: 173-194.

Kalt, E., R. Monfared, and M. Jackson. 2016. "Towards an Automated Polishing System - Capturing Manual Polishing Operations". International Journal of Research in Engineering and Technology 5: 182-192.

Khan, A.M., D. W. Yun, K. M. Zuhaib, J. Iqbal, R. J. Yan, F. Khan, and C. Han. 2017. "Estimation of desired motion intention and compliance control for upper limb assist exoskeleton". International Journal of Control, Automation and Systems 15: 802-814.

Kirschner, D., R. Velik, S. Yahyanejad, M. Brandstotter, and M. Hofbaur. 2016. "YuMi, Come and Play with Me. A Collaborative Robot for Piecing Together a Tangram Puzzle". Ronzhin A., Rigoll G., Meshch- 
eryakov R. (eds) Interactive Collaborative Robotics. ICR. Lecture Notes in Computer Science, vol. 9812. Springer.

Mohammad, A.E.K., J. Hong, and D. Wang. 2018. "Design of a force-controlled end-effector with lowinertia effect for robotic polishing using macro-mini robot approach". Robotics and Computer-Integrated Manufacturing 49: 54-65.

Nagata, F., T. Hase, Z. Haga, M. Omoto, and K. Watanabe. 2007. "CAD/CAM-based position/force controller for a mold polishing robot". Mechatronics 17 (4-5): 207-216.

Nakamura, Y., H. Hanafusa, and T. Yoshikawa. 1987. "Task-priority based redundancy control of robot manipulators". The Int. Journal of Robotics Research 6: 3-15.

Oba, Y., Y. Yamada. 2017. "Simultaneous tool posture and polishing force control of unknown curved surface using serial-parallel mechanism polishing machine". Precision Engineering 49: 24-32.

Ries, E. 2009. "What is the Minimum Viable Product". March. http://venturehacks.com/articles/minimumviable-product. 2009 (Accessed August, 2018).

Robinson, F. 2001 "A Proven Methodology to Maximize Return on Risk". http://www.syncdev.com/minimum-viable-product. (Accessed August, 2018).

Shepherd, S., and A. Buchstab. "KUKA Robots On-Site". McGee W., Ponce de Leon M. (eds) Robotic Fabrication in Architecture, Art and Design 2014. Springer.

Siciliano, B., and J. Slotine. 1991. "A general framework for managing multiple tasks in highly redundant robotic systems. Proceedings of the Fifth Int. Conference on Advanced Robotics (ICAR91), Pisa, Italy, 1991, pp. 1211-1216.

Surdilovic, D., and G. Schreck. 2010. "Development of collaborative robots (cobots) for flexible human integrated assembly automation". 41st International Symposium on Robotics and 6th German Conference on Robotics (ROBOTIK), 2010.

SYMPLEXITY: Symbiotic Human-Robot Solutions for Complex Surface Finishing Operations. European project funded by E.U. through the H2020. Project no. 637080. Call: H2020-FoF-2014. Topic: FoF-062014. Starting date: 01/01/2015. Duration: 48 months. https://www.symplexity.eu/ (Accessed March, 2019).

Vihlborg, P., I. Bryngelsson, B. Lindgren, L.G. Gunnarsson, and P. Graff. 2017. "Associatio between vibration exposure and hand-arm vibration symptoms in a Swedish mechanical industry". February 2017.

Vogel, J., S. Haddadin, B. Jarosiewicz, J. Simeral, D. Bacher, L. Hochberg, J. Donoghue, and P. van der Smagt. 2015. "An assistive decision-and-control architecture for force-sensitive hand-arm systems driven by human-machine interfaces". The International Journal of Robotics Research 34: 763-780. 